\title{
Managing patient expectations when
}

\section{a lockdown is mandated to reduce the}

spread of COVID-19

\section{Len D'Cruz}

Head of BDA Indemnity

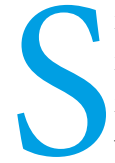

ince dental practices re-opened in early June, following the first national lockdown, dental teams have worked hard to clear the backlog of incomplete treatments and urgent care for existing patients, as well as coping with an additional demand from other patients whose own practice has limited capacity.

Millions of dental appointments were cancelled during the initial lockdown period and practices have since reorganised services and opening times to accommodate those patients whilst complying with national guidance on social distancing and the need for fallow time following aerosol generating procedures (AGPs).

Patients expect a dental practice to be COVID-safe with its adherence to social distancing in waiting rooms, screens at reception, hand sanitising facilities and appropriate PPE. And the GDC has already had cases before them regarding the lack of appropriate COVID-19 pandemic precautions ${ }^{1}$ that should have been provided by the dentist who owned the practice. The issue at stake here is the importance attached to public trust in the dental profession to uphold proper standards of conduct and behaviour.

Practices are advised to maintain a record of the standard operating procedure (SOP) with version control, chronicling the changes in practice as new guidance is issued by the four devolved health authorities or new peer reviewed evidence appears. The BDA 'Return to Practice toolkit" is a good place to find out just what should be in place.

Dentists and their team should record relevant COVID prevention details in the clinical record - such as whether an AGP was performed, what PPE was worn by the dental team in that room, fallow time and additional mitigating factors such as using rubber dam, high volume suction or mouthwashes for example.

If the different surgeries in the practice require different fallow times this should be noted in the SOP adopted by the practice together with any other procedures to manage this effectively and consistently. Where dentists, therapists and hygienists are working between surgeries this should be recorded in a daily rota and timetable, so that they can adopt the correct fallow time, which is also documented in the rota.

\section{Access to NHS care}

Patients are increasingly frustrated about their inability to access dental care. Media coverage and investigative reporting is now examining the disparity in care being provided across the country. These reports are particularly sensitive to situations where patients requesting NHS care are told it is unavailable but that private services are.

There may be completely legitimate reasons why such a situation arose, but it is important that dentists do not breach NHS contract regulations or the professional ethical standards set down by the regulator.

\section{GDC Standards for the dental team²} 1.7 .3

You must not mislead patients into believing that treatments which are available on the NHS (or equivalent health service) can only be provided privately. If you work in a purely private practice, you should make sure that patients know this before they attend for treatment.

\subsection{4}

If you work in a mixed practice, you must not pressurise patients into having private treatment if it is available to them under the
NHS (or equivalent health service) and they would prefer to have it under the NHS (or equivalent health service).

If a practice decides to charge patients the equivalent NHS charge for any treatment provided privately, the patient is entitled to know that this is under a private contract and that all the conditions and guarantees that apply to the NHS services will not therefore be underwritten by the NHS for that course of treatment.

To neglect to advise patients they are actually having a non-NHS course of treatment and the patient charges are not being collected for the NHS might be considered to be misleading. It would certainly be misleading and fraudulent for the patient to sign an NHS acceptance form and for no claim to be submitted for that course of treatment.

Trust is a distinctive quality of the relationship that exists between the dental profession and their patients. It can easily be undermined by media stories that suggest unprofessional and unethical behaviour which also make it harder for other practices to maintain the highest professional standards in very challenging circumstances.

The dental profession may be subject to additional episodes of precautionary restrictions over the next six months. By maintaining the trust of our patients from an early stage, the profession is best placed to manage any irritating frustrations that might lie ahead for those same patients, if further appointments have to be delayed or cancelled.

\section{References}

1. GDC Outcomes and Appeals list $10 \mathrm{C}$ interim Orders committee CAS-195102.

2. General Dental Council. Standards for the Dental Team. Available online at: https:// standards.gdc-uk.org/Assets/pdf/Standards $\% 20$ for\%20the\%20Dental\%20Team.pdf (Accessed September 2020).

https://doi.org/10.1038/s41404-020-0536-z 